\title{
Deterministic models of the simplest chemical reactions
}

\author{
Leonid A. Bunimovich
}

Mark Demers

Fairfield University, mdemers@fairfield.edu

Follow this and additional works at: https://digitalcommons.fairfield.edu/mathandcomputersciencefacultypubs

Copyright 2005 Springer-Verlag

The final publication is available at www.springerlink.com.

\section{Peer Reviewed}

\section{Repository Citation}

Bunimovich, Leonid A. and Demers, Mark, "Deterministic models of the simplest chemical reactions" (2005). Mathematics Faculty Publications. 41.

https://digitalcommons.fairfield.edu/mathandcomputerscience-facultypubs/41

\section{Published Citation}

Leonid A. Bunimovich and Mark Demers, "Deterministic models of the simplest chemical reactions," Journal of Statistical Physics 120 (2005), 239-252.

This Article is brought to you for free and open access by the Mathematics Department at DigitalCommons@Fairfield. It has been accepted for inclusion in Mathematics Faculty Publications by an authorized administrator of DigitalCommons@Fairfield. For more information, please contact digitalcommons@fairfield.edu. 


\title{
Deterministic Models of the Simplest Chemical Reactions
}

\author{
Leonid A. Bunimovich* Mark F. Demers ${ }^{\dagger}$
}

March 19, 2005

\begin{abstract}
We present a general mathematical framework for constructing deterministic models of simple chemical reactions. In such a model, an underlying dynamical system drives a process in which a particle undergoes a reaction (changes color) when it enters a certain subset (the catalytic site) of the phase space and (possibly) some other conditions are satisfied. The framework we suggest allows us to define the entropy of reaction precisely and does not rely, as was the case in previous studies, on a stochastic mechanism to generate additional entropy. Thus our approach provides a natural setting in which to derive macroscopic chemical reaction laws from microscopic deterministic dynamics without invoking any random mechanisms.
\end{abstract}

\section{Introduction}

The derivation of macroscopic chemical rate laws from microscopic deterministic dynamics is one of the central problems of statistical mechanics. To date there have been several attempts to establish this link by modeling chemically reacting processes using low-dimensional dynamical systems. In one dimension, Elskens, Frisch and Nicolis [EFN] studied non-interacting particles of two colors which change color when their trajectories cross. In [E] this model of isomerization kinetics was modified to allow for interacting particles. In two dimensions, coloring processes driven by the dynamics of triadic baker maps on chains of squares $([\mathrm{EK}],[\mathrm{GK}])$ have been studied and reaction-diffusion equations derived for the color densities.

Recently, the more realistic model of a two-dimensional Lorentz gas has been used to drive the underlying dynamics of the coloring system. In this model, a subset of the scatterers is chosen to be the reactive catalytic site. A particle, initially colored one of two colors, say red or green, is subject to the usual dynamics induced by elastic collisions with

${ }^{*}$ School of Mathematics and Center for Nonlinear Sciences, Georgia Institute of Technology, Atlanta GA 30332. Email: bunimovh@math.gatech.edu.

${ }^{\dagger}$ School of Mathematics, Georgia Institute of Technology, Atlanta GA 30332. Email: demers@math.gatech.edu. 
the fixed scatterers until it reaches the catalytic site. The particle changes color upon impact with the catalyst and continues to reflect elastically. In this setting, Nielsen and Kapral [NK] defined a colored entropy for the system which they related numerically to a colored rate of escape. Using their definition of colored entropy, the authors obtained what they described as surprising and counterintuitive results which we address in this paper. Lemma 2.1 and the example of Section 3.2 include the case considered in their paper and we reinterpret the entropy of such a system in the exact mathematical framework presented in Section 2.

Gaspard and Claus ([CG1], [CG2]) studied the reactive eigenmodes which govern the rate of relaxation of the color densities and derived the related reaction-diffusion equations. In order to obtain an increase in entropy due to the coloring process, however, they introduced a purely random mechanism which allowed the change in color to take place with probability $p$ each time the particle collided with the catalytic site.

The purpose of this paper is to present a general and purely deterministic mathematical setting for chemical reactions in dynamical systems. The corresponding mathematical model can be expressed as a skew product of measurable mappings. From this point of view, questions of deterministic chemical reactions in dynamical systems and especially of the entropy of such reactions are addressed rigorously and in a purely dynamical setting without invoking any probabilistic mechanisms. The coloring entropy of a general system is exactly defined and several basic examples are presented which demonstrate the different types of behavior of coloring systems. Although the examples we present in Section 3 use the Lorentz gas as the underlying dynamical system driving the motion of the colored particle, our approach, which we formulate in Section 2, is general and can be used for any underlying dynamical system. In an effort to make the paper self-contained, we include all necessary definitions and results.

\section{The Coloring System as a Skew Product}

Suppose we have a dynamical system in a space $X$ generated by a finite dimensional system of differential equations or a map. We place a particle at a point in $X$ and attach to that particle a color, red or green. We choose a subset $C$ of $X$ which we refer to as the catalytic site for the coloring reaction. We then consider the orbit of the particle under the dynamics in $X$. The color of the particle changes from red to green or vice versa only when the particle enters the catalytic site $C$; however, we may also consider additional conditions which must be met in order for the color change to take place. We model these via a function acting on a space $Y$ representing variables which influence the chemical reaction.

The additional condition expressed by the parameter $y \in Y$ can be interpreted in a number of ways depending on the specific model under consideration. In the example of Section 3.2 we consider it as a threshold which must be exceeded in order to trigger the chemical reaction at the catalytic site. In Section 3.3, y represents the velocity of the particle and in Section 3.4 it represents the (unknown) dynamics of a second particle in the system. In general, $y$ will represent a component of the system (possibly depending 
on the dynamics in $X$ ) which affects the coloring process.

We formulate our approach as follows. We assume that $(X, \mathcal{A})$ and $(Y, \mathcal{B})$ are Lebesgue spaces with probability measures $\mu_{X}$ and $\mu_{Y}$ and that $T: X \rightarrow X$ is a measure-preserving transformation of $X$. Let $\left\{S_{x}\right\}_{x \in X}$ be a measurable family of transformations of $Y$ which preserve the measure $\mu_{Y}$. We call $X$ the base of the skew product and refer to the copy of $Y$ above each $x \in X$ as a fiber.

The catalytic site $C$ is a measurable subset of $X$. We denote the color of the particle by $\alpha= \pm 1$ and assume for simplicity that the additional condition introduced above can be expressed in terms of a (possibly vector) parameter $y$ belonging to a measurable set $D \subset Y$. The coloring system can be expressed simply as the skew product

$$
U(x, y, \alpha)=\left(T x, S_{x} y, R_{x, y} \alpha\right)
$$

where $R_{x, y} \alpha=\alpha^{\prime}$ is different from $\alpha$ if and only if $T x \in C$ and $S_{x} y \in D$. We define $Z=\{-1,1\}$ and note that $R_{x, y}: Z \circlearrowleft$ is a measurable family of maps on $X \times Y$.

\subsection{Rate of Reaction}

Reaction rates for models of chemical reactions can be defined in several ways. In this paper, we are concerned with models in which a single particle changes color over time, so it is natural to consider an average rate of reaction rather than an instantaneous one. In this section, we define the asymptotic rate of reaction in order to discuss its relation to the coloring entropy defined in Section 2.3. This relation will be discussed in the examples of Section 3.

The asymptotic rate of reaction $\rho$ of the coloring system (1) can be expressed as the average of the asymptotic rates along orbits. Letting $\chi_{A}$ denote the characteristic function of a set $A \subset X \times Y$, we define

$$
\rho:=\lim _{n \rightarrow \infty} \frac{1}{n} \int_{X} \int_{Y} \sum_{i=1}^{n} \chi_{C \times D}\left(T^{i} x, S_{x}^{i} y\right) d \mu_{Y} d \mu_{X}
$$

where $S_{x}^{i}$ denotes the iterates of $S_{x}$ along the orbit of $x$, i.e., $S_{x}^{i} y=S_{T^{i-1} x} \cdots S_{T x} S_{x} y$. When the skew product $\left(T, S_{x}\right)$ is ergodic, the averages $\frac{1}{n} \sum_{i=1}^{n} \chi_{C \times D}\left(T^{i} x, S_{x}^{i} y\right)$ converge to a constant so that $\rho$ takes on an especially simple form,

$$
\rho=\mu_{X}(C) \mu_{Y}(D) .
$$

We will use these descriptions of the reaction rate in discussing the examples of Section 3 , two of which are ergodic and one of which is not. In general, reaction rates for non-ergodic systems behave in a complex manner and the usual statistical arguments do not hold. See for example, [DB].

\subsection{Abramov-Rokhlin Formula}

We recall the Abramov-Rokhlin formula for the entropy of a skew product [AR]. Given Lebesgue spaces $(X, \mathcal{A})$ and $(Y, \mathcal{B})$ with probability measures $\mu_{X}$ and $\mu_{Y}$, define $W=$ 
$X \times Y$ and $\lambda=\mu_{X} \otimes \mu_{Y}$. Let $T$ and $\left\{S_{x}\right\}_{x \in X}$ be defined as above. The skew product $U(x, y)=\left(T x, S_{x} y\right)$ is a measurable transformation on $X \times Y$ and the measure-theoretic entropy of $U$ with respect to $\lambda$ is given by

$$
h(U)=h(T)+h_{T}(S)
$$

where $h(T)$ is the entropy of $T$ with respect to $\mu_{X}$ and $h_{T}(S)$ is defined as follows. For any partition $\eta$ of $Y$ with finite entropy $H(\eta)$, define $\eta_{x}^{n}=\bigvee_{k=0}^{n-1} S_{x}^{-1} S_{T x}^{-1} \cdots S_{T^{k-1} x}^{-1} \eta$. Then $h_{T}(S, \eta)=\inf _{n} \frac{1}{n} \int_{X} H\left(\eta_{x}^{n}\right) d \mu_{X}(x)$ and $h_{T}(S)=\sup _{\eta} h_{T}(S, \eta)$.

When $U=U(x, y, \alpha)=\left(T x, S_{x} y, R_{x, y} \alpha\right)$ as in the case of system (1), the formula generalizes to $h(U)=h(T)+h_{T}(S)+h_{S, T}(R)$. In our case, however, $h_{S, T}(R)=0$ since $Z$ is a discrete space with only two points.

\subsection{Coloring Entropy}

Let $T, S, R$ and $U$ be as defined in the description of the coloring system (1). Let $\xi=\{D, Y \backslash D\}$ be the partition of $Y$ defined by the coloring condition $R_{x, y} \alpha=-\alpha$ if and only if $\left(T x, S_{x} y\right) \in C \times D$. Following (4), we define the coloring entropy of such a system to be

$$
h_{c}(U)=h_{T}(S, \xi) .
$$

This definition of coloring entropy is motivated by considering the sequence of maps $R_{x, y}^{n} \alpha=R_{T^{n-1} x, S_{x}^{n-1} y} \cdots R_{x, y} \alpha$ which either change or do not change the color of the particle as the system evolves. The entropy that we have defined measures the uncertainty inherent in predicting the color $\alpha_{n}$ of the particle at time $n$, knowing only the orbit of the coordinate $x$ and its coloring history $\alpha_{0}, \ldots, \alpha_{n-1}$. This is precisely the information contained in the map $T$ and the partition $\xi$.

At first glance, one might be tempted to define the coloring entropy simply as $h_{T}(S)$ since that is the difference between the entropy of the underlying dynamical system $h(T)$ and that of the skew product $h(U)$. However, $h_{T}(S)$ does not reflect any information about $D$, which clearly affects the rate (and entropy) of the coloring process. In fact, if $D$ is the empty set, no coloring occurs and there should be no coloring entropy.

Our first observation is along these lines and is fairly straightforward.

Lemma 2.1 If $U=U(x, \alpha)$, i.e. $\mathrm{U}$ does not depend on an additional parameter $y$, then the coloring entropy is zero.

This lemma says that if we take the case in which the particle changes color if and only if it enters a certain subset $C$ of $X$, then there is no increase in entropy due to the coloring process. In fact, this is precisely the setting of $[\mathrm{NK}]$ in which the authors attempted to compute a kind of colored entropy. Given the mathematical framework presented here, it is clear that there can be no increase in entropy due to the coloring process without the introduction of an additional parameter to control the reaction. 
Proof of Lemma. If $U=U(x, \alpha)$, we can put the system into the form of (1) by artificially introducing $y$. Simply let $S$ be any measure-preserving map of a Lebesgue space $Y$ and set $D=Y$ or $\emptyset$. In either case, $\xi$ is the trivial partition so that $h_{T}(S, \xi)=0$.

It is possible to construct a coloring condition so that $D=D_{x}$ varies according to $x$ (see the second and third examples of Section 3). Now $\xi=\xi_{x}$ depends on $x$ and we need to return to the motivation provided by the coloring sequence $\alpha_{0}, \ldots, \alpha_{n-1}$ in order to interpret (5) in this case. Knowing $\alpha_{n}$ given $\alpha_{0}, \ldots, \alpha_{n-1}$ means knowing whether $S_{T^{n-1} x} \cdots S_{T x} S_{x} y \in D_{T^{n} x}$ or not. This is the information contained in the partition $S_{x}^{-1} S_{T x}^{-1} \cdots S_{T^{n-1} x}^{-1} \xi_{T^{n} x}$. Putting this together with the definitions of Section 2.2, we see that

$$
H\left(\xi_{x}^{n}\right)=H\left(\bigvee_{k=0}^{n-1} S_{x}^{-1} S_{T x}^{-1} \cdots S_{T^{k-1} x}^{-1} \xi_{T^{k} x}\right)
$$

is the information known at the $n^{\text {th }}$ step so that

$$
h_{T}(S, \xi)=\lim _{n \rightarrow \infty} \frac{1}{n} \int_{X} H\left(\bigvee_{k=0}^{n-1} S_{x}^{-1} S_{T x}^{-1} \cdots S_{T^{k-1} x}^{-1} \xi_{T^{k} x}\right) d \mu_{X} .
$$

With this interpretation of $H\left(\xi_{x}^{n}\right)$, the definition (5) remains the same.

It is a standard result of ergodic theory that the limit in (6) exists as long as the family of partitions $\left\{\xi_{x}\right\}$ depends measurably on $x$ and $\int_{X} H\left(\xi_{x}\right) d \mu_{X}<\infty$. Indeed, letting $f_{n}(x)=H\left(\bigvee_{k=0}^{n-1} S_{x}^{-1} S_{T x}^{-1} \cdots S_{T^{k-1} x}^{-1} \xi_{T^{k} x}\right)$, it is easy to show that $f_{n+m}(x) \leq$ $f_{n}(x)+f_{m}\left(T^{n} x\right)$ for each $n, m \geq 0$ so that $\lim _{n \rightarrow \infty} \frac{1}{n} f_{n}(x)$ exists for almost every $x$ by the subadditive ergodic theorem (see for instance [W, $\S 10.2]$ ).

The definition of the reaction rate $\rho$ is also modified in the obvious way so that equation 2 becomes

$$
\rho=\lim _{n \rightarrow \infty} \frac{1}{n} \int_{X} \int_{Y} \sum_{i=1}^{n} \chi_{C \times D_{T^{i} x}}\left(T^{i} x, S_{x}^{i} y\right) d \mu_{Y} d \mu_{X} .
$$

In this case, when $\left(T, S_{x}\right)$ is ergodic, although the simple expression (3) is no longer valid, the ergodic averages do converge to a constant,

$$
\rho=\int_{X} \int_{Y} \chi_{C \times D_{x}} d \mu_{Y} d \mu_{X}
$$

\section{Deterministic Dynamical Coloring Models}

In this section, we present three examples of coloring models in order to give some intuition for how the mathematical framework of the previous section applies to coloring systems. The examples presented below capture three typical possibilities for a general coloring system: zero coloring entropy, positive coloring entropy with a known mechanism, and a particle in a thermostat in which the unknown dynamics of other particles affect the coloring process. 


\subsection{Setting}

For simplicity, in each of the following examples our underlying dynamical system is a Lorentz gas on a torus reflecting elastically off three convex scatterers of equal size arranged symmetrically. The flow moves with constant speed and we let $\tau_{\text {min }}$ be the minimum time required between collisions. We assume the system has finite horizon and denote this maximum time between collisions by $\tau_{\max }$.

The billiard map induced by the flow is given in canonical coordinates as $T(i, s, \varphi)=$ $\left(i^{\prime}, s^{\prime}, \varphi^{\prime}\right)$ where $i$ is the scatterer, $s$ is the position on the scatterer, $0 \leq s \leq 2 \pi$, and $\varphi$ is the angle made with the normal vector on the scatterer after reflection, $-\pi / 2 \leq \varphi \leq \pi / 2$. Set $x=(i, s, \varphi)$ and let $X$ denote the domain of $T$. We refer to the angular coordinate of a point $x \in X$ by $\varphi(x)$. Let $\mu$ be the usual absolutely continuous invariant measure for the billiard map given by $d \mu=c \cos \varphi d \varphi d s$ where $c$ is the normalizing constant.

We choose one of the three scatterers to be the catalytic site where our reaction will take place. For definiteness, let us say the catalytic scatterer $C$ is $i=1$.

\subsection{A Simple Example with Positive Coloring Entropy}

We build the skew-product $U$ by introducing the parameter space $Y=[0,1]$ and the doubling map $f(y)=2 y(\bmod 1)$. Let $S_{x}=f$ if $T x \in C$ and $|\varphi(T x)| \leq a ; S_{x}=I d$ otherwise. The choice of $0 \leq a \leq \frac{\pi}{2}$ is arbitrary, but the added condition on $x$ models a reaction which requires a sufficiently direct collision of the particle with the coloring site. A collision too close to tangential does not permit a color change to take place. (This is reminiscent of enzyme docking and the steric effect in the study of protein bonding $[\mathrm{HMWN}])$. Let $C^{\prime}=\{x \in C:|\varphi| \leq a\}$.

We choose $L$ between 0 and 1 and define $D=[L, 1]$. Then the coloring map $R_{x, y}$ is defined as

$$
R_{x, y} \alpha=\left\{\begin{array}{cl}
-\alpha & , \text { if } T x \in C^{\prime} \text { and } f(y) \in D \\
\alpha & , \text { otherwise }
\end{array}\right.
$$

where as before $\alpha= \pm 1$ represents the color of the particle. The number $L$ which defines the condition on $y$ for the color to change is suggestive of a threshold which must be exceeded in order for the reaction to take place.

Using the notation of Section 2 for this example, $\mu_{X}=\mu$ and $\mu_{Y}$ is Lebesgue measure on $[0,1]$ since both $f$ and $I d$ preserve Lebesgue measure. Given $x \in X$, let $j_{n}(x)=\#\{0 \leq$ $\left.i<n: T^{i}(x) \in C^{\prime}\right\}$. Then

$$
\begin{aligned}
\frac{1}{n} H\left(\xi_{x}^{n}\right) & =\frac{1}{n} H\left(\bigvee_{k=0}^{n-1} S_{x}^{-1} S_{T x}^{-1} \cdots S_{T^{k-1} x}^{-1} \xi\right) \\
& =\frac{1}{n} H\left(\bigvee_{k=0}^{j_{n}(x)} f^{-k} \xi\right) \\
& =\frac{j_{n}(x)}{n} \frac{1}{j_{n}(x)} H\left(\bigvee_{k=0}^{j_{n}(x)} f^{-k} \xi\right) .
\end{aligned}
$$


For $\mu$-almost-every $x, \frac{j_{n}(x)}{n} \rightarrow \mu\left(C^{\prime}\right)$ since $T$ is ergodic and $\frac{1}{j_{n}(x)} H\left(\bigvee_{k=0}^{j_{n}(x)} f^{-k} \xi\right) \rightarrow h(f, \xi)$ as $n \rightarrow \infty$. Now $\mu\left(C^{\prime}\right)=\frac{1}{3} \sin a$ so that

$$
h_{c}=h_{T}(S, \xi)=\frac{\sin a}{3} h(f, \xi)
$$

The entropy is maximized when $L=\frac{1}{2}$ and in this case $h(f, \xi)=\log 2$. In general, $h(f, \xi) \leq H(\xi)=L \log L+(1-L) \log (1-L)$ which shows that the coloring entropy vanishes as it should when the level $L$ approaches 0 (a reaction always occurs when $x \in C^{\prime}$ ) or 1 (no coloring reaction can occur). The same is true when the angle with the normal required for a sufficiently direct collision is decreased to zero.

This simple example also illuminates the relationship between the coloring entropy and the asymptotic rate of reaction defined earlier. Since $\left(T, S_{x}\right)$ is ergodic, we may use equation (3) together with (7) to write

$$
h_{c}=\mu_{X}\left(C^{\prime}\right) h(f, \xi)=\frac{\rho}{\mu_{Y}(D)} h(f, \xi) .
$$

The process obviously needs a positive asymptotic reaction rate to generate coloring entropy, but it needs something stronger: positive entropy generated by the map on the fiber. This illustrates the fact that reaction rates are concerned with ergodicity while coloring entropy requires stronger chaotic properties (e.g. nonvanishing Lyapunov exponents on fibers).

\subsection{An Example with Zero Coloring Entropy}

We now introduce an example of a coloring process based on the Lorentz gas that has an element of uncertainty, but for which the coloring entropy is zero. We will later modify the example to obtain a related system with positive coloring entropy. We color a particle in the Lorentz gas red or green. In this example we do not assume that the particle moves with unit speed. When the particle collides with the catalyst, its color changes if and only if the particle has collided with the catalyst at least $k$ times in a given time interval of length $\tau_{*}$ before the present collision. We think of this condition of color change as requiring the catalyst to be "sufficiently excited" before it reacts with the particle. Although the reaction needs information inherited from the flow, not just the billiard map, the coloring process yields no increase in entropy.

Let $\tau(x)$ denote the time until the next collision for a particle starting at $x$ and moving under the flow at constant speed. Set $t_{0}=0$ and let $t_{n}=\sum_{i=0}^{n-1} \tau\left(T^{i} x\right)$ denote the time of the $n^{\text {th }}$ collision starting at $x$ for $n \geq 1$.

A point in the coloring system is given by $(x, \alpha)$ where $x$ is as before and $\alpha= \pm 1$ denotes the color of the particle. We allow the system to flow for at least $n>\tau_{*} / \tau_{\text {min }}$ iterates so that we may take sufficiently many preimages of a given point in the catalytic site. The dynamics of the system are given by the coloring billiard map $U$,

$$
U(x, \alpha)=\left(T x, R_{x} \alpha\right)
$$


where $R_{x}$ is defined by

$$
R_{x} \alpha= \begin{cases}-\alpha, & \text { if } T x \in C \text { and the catalyst has been hit at least } \\ & k \text { times in the preceding time interval of length } \tau_{*} \\ \alpha, & \text { otherwise }\end{cases}
$$

We are free to choose $k$ and $\tau_{*}$ according to how often we want the reaction to occur.

In order to codify this condition more precisely, define $r(x)=\max \left\{j: t_{j}\left(T^{-j} x\right) \leq \tau_{*}\right\}$. The function $r(x)$ represents the number of collisions undergone by the trajectory ending at $x$ in the preceding interval of time $\tau_{*}$ under the flow. Note that $\tau_{*} / \tau_{\max } \leq r \leq \tau_{*} / \tau_{\min }$ once the system has run for at least time $\tau_{*}$. Let $m(x)=\#\left\{-r(x) \leq i \leq 0: T^{i} x \in C\right\}$ and define $C_{v}^{\prime}=\{x \in C: m(x) \geq k\}$. Then the condition for coloring becomes

$$
R_{x} \alpha=-\alpha \text { if and only if } T x \in C_{v}^{\prime} .
$$

The only uncertainty here is given by the magnitude $v$ of the velocity of the particle which is not known and which clearly affects the set $C_{v}^{\prime}$.

We can formalize this from the point of view of the skew product in the following way. Let $Y$ be the space of permissible speeds and let $\mu_{Y}$ be any probability measure on $Y$. If $x \in C$, we let $D_{x}=\left\{y \in Y: x \in C_{y}^{\prime}\right\}$, i.e. $D_{x}$ is the set of speeds for which the orbit of $x$ would have collided with the catalyst sufficiently often to trigger a reaction. Then the full coloring system becomes

$$
U(x, y, \alpha)=\left(T x, y, R_{x, y} \alpha\right)
$$

where $R_{x, y} \alpha=-\alpha$ if and only if $(T x, y) \in C \times D_{T x}$. Note that $S_{x}=I d_{Y}$ since the velocity remains constant. Using (6), we see that $h_{c}=h_{T}(I d, \xi)=0$.

Although the direct product $\left(T, I d_{Y}\right)$ is not ergodic, we can still write down an expression for the asymptotic rate of reaction which depends on the measure $\mu_{Y}$ we choose for the velocities.

$$
\begin{aligned}
\rho & =\lim _{n \rightarrow \infty} \int_{X} \int_{Y} \frac{1}{n} \sum_{i=1}^{n} \chi_{C \times D_{T^{i} x}}\left(T^{i} x, y\right) d \mu_{Y} d \mu_{X} \\
& =\lim _{n \rightarrow \infty} \int_{Y} \int_{X} \frac{1}{n} \sum_{i=1}^{n} \chi_{C_{y}^{\prime}}\left(T^{i} x\right) d \mu_{X} d \mu_{Y}
\end{aligned}
$$

For fixed $y$, the sum converges by the ergodic theorem to $\mu_{X}\left(C_{y}^{\prime}\right)$ since $\left(T, \mu_{X}\right)$ is ergodic. This yields two equivalent expressions for the reaction rate,

$$
\rho=\int_{Y} \mu_{X}\left(C_{y}^{\prime}\right) d \mu_{Y}=\int_{X} \mu_{Y}\left(D_{x}\right) d \mu_{X}
$$

If one wishes to consider the velocity of the particle fixed at a certain speed $y$ with no uncertainty, then the rate becomes simply $\rho=\mu_{X}\left(C_{y}^{\prime}\right)$. 


\subsection{The Modified System with Positive Coloring Entropy}

It is possible to modify the preceding example so that it does yield positive coloring entropy. The idea is to consider a tagged particle in a Lorentz gas of noninteracting particles. Only the position and velocity of the tagged particle are known. For simplicity, we assume here only the existence of a second particle which must help to excite the catalyst. We take $T, X$ and $\mu$ as before. In order to synchronize time, it is convenient to let $\left(S_{t}, Y\right)$ be the billiard flow (rather than another billiard map) on the torus with the same configuration of scatterers which induces the billiard map $T$. We fix the speed of both particles and form the standard skew product $V(x, y)=\left(T x, S_{\tau(x)} y\right)$, recalling that $\tau(x)$ represents the free flight time from $x$ to $T x$ under the flow. The state space $X \times Y$ describes the Lorentz gas with two non-interacting particles and we keep track of only the particle $x$ and its coloring history. The trajectory of the other particle $y$ is unknown.

We let $\tilde{C} \subset Y$ be the catalytic scatterer $i=1$ for the particle $y$; this corresponds to the catalytic site $C \subset X$ for $x$. Define $q(y)=\#\left\{-\tau_{*} \leq t \leq 0: S_{t} y \in \tilde{C}\right\}$. The coloring system then becomes $U(x, y, \alpha)=\left(T x, S_{\tau(x)} y, R_{x, y} \alpha\right)$ where the coloring map is given by

$$
R_{x, y} \alpha=\left\{\begin{array}{cl}
-\alpha, & \text { if } T x \in C \text { and } m(T x)+q\left(S_{\tau(x)} y\right) \geq k \\
\alpha, & \text { otherwise }
\end{array} .\right.
$$

For $x \in C$, set $D_{x}=\{y \in Y: q(y) \geq k-m(x)\}$ while for $x \notin C, D_{x}=\emptyset$. This defines the family of partitions $\xi_{x}$ so that coloring occurs after one iterate if and only if $T x \in C$ and $S_{\tau(x)} y \in D_{T x}$. This yields

$$
\xi_{x}^{n}=\bigvee_{i=0}^{n-1} S_{\tau(x)}^{-1} S_{\tau(T x)}^{-1} \cdots S_{\tau\left(T^{i-1} x\right)}^{-1} \xi_{T^{i} x}=\bigvee_{i=0}^{n-1} S_{-t_{i}(x)} \xi_{T^{i} x}
$$

where as before $t_{i}(x)=\sum_{j=0}^{i-1} \tau\left(T^{j} x\right)$. Note that $\xi_{x}$ is the trivial partition when $x \notin C$ so that

$$
\xi_{x}^{n}=\bigvee_{\ell=0}^{j_{n}(x)} S_{-t_{i_{\ell}}(x)} \xi_{T^{i} \ell(x)}
$$

where $i_{\ell}$ are the integers $i$ when $T^{i} x \in C$ and $j_{n}(x)=\#\left\{0 \leq i<n: T^{i} x \in C\right\}$. As in the first example, this yields

$$
\begin{aligned}
h_{c} & =\lim _{n \rightarrow \infty} \int_{X} \frac{1}{n} H\left(\xi_{x}^{n}\right) d \mu(x)=\lim _{n \rightarrow \infty} \int_{X} \frac{j_{n}(x)}{n} \frac{1}{j_{n}(x)} H\left(\bigvee_{\ell=0}^{j_{n}(x)} S_{-t_{i_{\ell}}(x)} \xi_{T^{i}(x)}\right) d \mu(x) \\
& \rightarrow \mu(C) \lim _{n \rightarrow \infty} \int_{X} \frac{1}{j_{n}(x)} H\left(\bigvee_{\ell=0}^{j_{n}(x)} S_{-t_{i_{\ell}}(x)} \xi_{T^{i} \ell(x)}\right) d \mu(x) .
\end{aligned}
$$

Note that if $k$ equals 0 or 1 , then $\xi_{x}$ is the trivial partition for all $x$, the coloring process is independent of the second particle $y$, and $h_{c}=0$. The same is true if the given time threshold $\tau_{*}$ is too short, namely if $\tau_{*}<k \tau_{\text {min }}$; then no coloring can occur, $\xi_{x}$ is again the trivial partition for all $x$, and $h_{c}=0$. 
However, with a suitable choice of $k$ and $\tau_{*}$, the coloring entropy is positive. To see this, note that the family of sets $D_{x}$ is really only a finite collection $D_{0}, \ldots, D_{k}$, where $D_{x}=D_{i}$ if $k-m(x)=i$ for $1 \leq i \leq k$ and $D_{x}=D_{0}=Y$ if $m(x) \geq k$. This means there are at most $k+1$ partitions being permuted in the expression for coloring entropy, $\xi_{0}, \ldots, \xi_{k}$. A suitable choice of $k$ and $\tau_{*}$ means that a positive measure set of $x$ and $y$ satisfy $m(x)+q(y) \geq k$. Without loss of generality, let us assume that the set $C_{1}=\{x \in C: m(x)=k-1\}$ has positive measure. For $x \in C_{1}, D_{x}=D_{1}$ and $\xi_{1}$ is not the trivial partition. So we can write

$$
\begin{aligned}
H\left(\bigvee_{\ell=0}^{j_{n}(x)} S_{-t_{i_{\ell}}(x)} \xi_{T^{i_{\ell}(x)}}\right) & =H\left(\bigvee_{\ell=0}^{j_{n}^{1}(x)} S_{-t_{i_{\ell}^{1}}(x)} \xi_{1} \vee \cdots \vee \bigvee_{\ell=0}^{j_{n}^{k}(x)} S_{-t_{i_{\ell}}(x)} \xi_{k}\right) \\
& \geq H\left(\bigvee_{\ell=0}^{j_{n}^{1}(x)} S_{-t_{i_{\ell}^{1}}(x)} \xi_{1}\right) .
\end{aligned}
$$

In the first step we have grouped the partitions according to whether $D_{x}=D_{i}$ and in the second step, we choose to focus on the entropy associated with one of these, namely $\xi_{1}$. The index $j_{n}^{1}(x)$ represents the number of times $T^{i} x \in C_{1}, 0 \leq i \leq n$, and $i_{\ell}^{1}$, $\ell=0, \ldots, j_{n}^{1}(x)$, are the times when $T^{i} x \in C_{1}$.

The expression (8) represents the entropy associated with a skew product of the form $\left(P x, S_{f(x)} y\right)$. In this example, $P$ is the first return map to $C_{1}$ and $f(x)=t_{i}(x)$ where $i$ is the smallest positive integer $i$ such that $T^{i} x$ returns to $C_{1}$. This type of skew product has been studied in some detail in the case when $f(x): C_{1} \rightarrow \mathbb{Z}^{+}$satisfies $f \geq \beta>0$ and $\int_{C_{1}} f d \mu_{C_{1}}<\infty$ (see $[\mathrm{N}]$ ). It was shown that such maps have conditional entropy $h_{P}\left(S_{1}\right)=h\left(S_{1}\right) \int_{C_{1}} f d \mu_{C_{1}}$. In our case, $h\left(S_{1}\right)>0$ since $S_{t}$ is the billiard flow, $f \geq \tau_{\text {min }}>$ 0 , and $\int_{C_{1}} f d \mu_{C_{1}}=\left(\mu C_{1}\right)^{-1} \int_{X} \tau d \mu<\infty$ so that $h_{P}\left(S_{1}\right)>0$. Since $S_{t}$ is a K-system ([S]) and $\xi_{1}$ is not the trivial partition, $h_{P}\left(S_{1}, \xi_{1}\right)>0$ as well, so that the coloring entropy is positive.

Observe that although the coloring entropy is produced by the second particle, it does not equal the entropy of a one-particle Lorentz gas. This would be the case if we considered two independently moving particles, i.e., a direct product of the corresponding dynamical systems. But there is a kind of interaction between particles in our model because the collisions of both particles with the catalytic site are counted and because the partitions $\xi_{x}^{n}$ of $Y$ are determined by the time between collisions of $x$, not $y$.

Since $\left(T, S_{x}\right)$ is ergodic, we easily calculate the asymptotic rate of reaction as

$$
\rho=\int_{X} \int_{Y} \chi_{C \times D_{x}} d \mu_{Y} d \mu_{X}=\int_{C} \mu_{Y}\left(D_{x}\right) d \mu_{X}
$$

This is the same as the expression for the rate in the previous example, although in that example the skew product is not ergodic and the system has zero coloring entropy. 


\section{Concluding Remarks}

In the above examples based on the Lorentz gas, we chose a configuration of three symmetrically arranged scatterers for simplicity. It is clear that the mathematical setting is valid for any configuration of convex scatterers on a torus with a bounded free path. The catalytic site $C$ need not be one of the scatterers, but may be taken to be a subset of one or several of them. In general of course, the base of the skew product need not be a Lorentz gas at all: the mathematical setting presented in Section 2 is general enough to include any dynamical system which is chosen to model the motion of the reacting particle.

The examples of Section 3 point out an important difference between reaction rates and coloring entropy. The former relies on the ergodicity of the skew product $\left(T, S_{x}\right)$ and the associated statistical properties of the system along orbits; the latter, on the other hand, requires that the dynamics on fibers of the skew product be chaotic in addition to the usual ergodicity in order to generate positive entropy. In fact, this is precisely why Lemma 2.1 holds. The space $Z=\{-1,1\}$ which represents the set of possible colors of the particle contains only two elements and so cannot generate any entropy. This is what necessitates the introduction of the additional parameter $y$ (the internal "chemical" variable) to control the color change and create the additional uncertainty necessary for positive coloring entropy.

In this paper we have given a general framework for constructing deterministic models of chemical reactions. Our approach is quite broad and allows us to construct more complicated models of concrete chemical reactions than have previously been available. Indeed the parameter $y$ could be a vector with any (finite) number of components. Alternatively, one can consider a skew product generated by any (finite) number of maps, i.e. $U\left(x, y_{1}, y_{2}, \ldots, y_{m}, \alpha\right)=\left(T(x), S_{x}\left(y_{1}\right), S_{x, y_{1}}\left(y_{2}\right), S_{x, y_{1}, \ldots, y_{m-1}}\left(y_{m}\right), R_{x, y_{1}, \ldots, y_{m}}(\alpha)\right)$ where all $y_{i}, 1 \leq i \leq m$, as well as $\alpha$, are vectors.

Moreover, our approach is consistently deterministic and does not require a stochastic mechanism (a random trial) to decide whether or not a reaction in fact occurs. Therefore, it provides a natural and consistently deterministic framework for the derivation of macroscopic chemical rate laws from microscopic deterministic dynamics.

Acknowledgments. We are indebted to Yu. Kifer for helpful discussions. The work of L.B. was partially supported by NSF grant \# DMS-0140165. M.D. was partially supported by NSF VIGRE grant \# DMS-0135290.

\section{References}

[AR] L.M. Abramov and V.A. Rokhlin, Entropy of Skew Product Mappings with Invariant Measures, Vestnik Leningrad Univ. 17:7 (1962), 5-13.

[CG1] I. Claus and P. Gaspard, Microscopic Chaos and Reaction-Diffusion Processes in the Periodic Lorentz Gas, J. Stat. Phys. 101 (2000), 161-186. 
[CG2] I. Claus and P. Gaspard, The Fractality of the Relaxation Modes in Deterministic Reaction-Diffusion Systems, Physica D 168-169 (2002), 266-291.

[DB] N. DeLeon and B.J. Berne, Intramolecular Rate Process: Isomerization Dynamics and the Transition to Chaos J. Chem. Phys. 75:7 (1981), 3495-3510.

[E] Y. Elskens, Microscopic Derivation of a Markovian Master Equation in a Deterministic Model of Chemical Reaction, J. Stat. Phys. 37 (1984), 673-695.

[EFN] Y. Elskens, H. Frisch and G. Nicolis, Exact Solution of a Deterministic Model for Isomerization Kinetics, J. Stat. Phys. 33 (1983), 317-339.

[EK] Y. Elskens and R. Kapral, Reversible dynamics and the macroscopic rate law for a solvable Kolmogorov system: the three bakers' reaction, J. Stat. Phys. $\mathbf{3 8}$ (1985), 1027-1049.

[GK] P. Gaspard and R. Klages, Chaotic and Fractal Properties of Deterministic Diffusion-Reaction Processes, Chaos 8:2 (1998), 409-423.

[HMWN] I. Halperin, B. Ma, H. Wolfson and R. Nussinov, Principles of Docking: An Overview of Search Algorithms and a Guide to Scoring Functions, Proteins: Structure, Function, and Genetics, 47 (2002), 409-443.

[N] D. Newton, On the Entropy of Certain Classes of Skew-Product Transformations, Proc. Amer. Math. Soc. 21:3 (1969), 722-726.

[NK] S. Nielsen and R. Kapral, Coloring a Lorentz Gas, J. Chem. Phys. 109:15 (1998), 6460-6468.

[S] Ya. G. Sinai, Dynamical Systems with Elastic Reflections. Ergodic Properties of Dispersing Billiards, Russian Math. Surveys 25:2 (1970), 137-189.

[W] P. Walters, An Introduction to Ergodic Theory, Springer-Verlag: New York, 1982. 